\title{
Clutter Modeling Based on SIRP Principle
}

\author{
Bin Wang ${ }^{1, a}$, Xin Song ${ }^{2, b}$ and Fengming Xin $^{3, c}$
}

${ }^{1,2,3}$ Northeastern University at Qinhuangdao, Taishan Road No. 143, Qinhuangdao, China

awangbinneu@qq.com, bxin78916@mail.neuq.edu.cn, xfm_neu@126.com

Keywords: intelligent radar, clutter modeling, SIRP, K distribution

\begin{abstract}
With the development of science and technology, more extensive use of electromagnetic spectrum will make radar environment become more and more complex. So radar should develop towards intelligent direction and clutter modeling needs to be more accurate. In this paper, based on SIRP principle, we research on mathematical model for $\mathrm{K}$ distribution clutter. Simulation results show the validity of our method. Finally, the whole paper is summarized.
\end{abstract}

\section{Introduction}

Radar echo generally contains two parts: useful radar target echo and radar clutter. The existence of clutter has great influences on the detection of radar target. With the development of space technology and continuous exploration of outer space, more extensive use of electromagnetic spectrum and high speed of target will make electromagnetic environment become more and more complex. The influence of clutter can not be ignored. In 2006, Simon Haykin firstly proposes the concept of intelligent radar, which is called cognitive radar and may adaptively and intelligently interrogate a propagation channel using all available knowledge[1].

Much work has been done in the related field. In [2], using curved wave spectral estimation that yields reliable results for any refractivity profile is suggested, in contrast to plane wave spectral estimation. In [3], the authors study the challenging problem of detecting a low radar cross-section target in heavy sea clutter by proposing a physics-based sea clutter generation model. In [4], the authors present a statistically non-Gaussian, spacetime clutter model in varying bistatic geometrical scenarios to validate the potential space-time adaptive processing algorithms for airborne bistatic radar clutter suppression. In [5], a new empirical model for sea clutter reflectivity at low grazing angles is developed. In [6], a sample of results from a statistical and spectral analysis of a set of sea spikes selected from the radar returns is shown. In [7], the authors propose a clutter model for such scanning radar applications taking the effect of scanning on clutter correlation into consideration.

In this paper, we mainly research on mathematical model for $\mathrm{K}$ distribution clutter based on SIRP principle.

\section{SIRP Principle Description}

In fact, clutter simulation is to generate a series of correlation random sequences which obey certain probability density distribution in amplitude. The basic thought of SIRP method is to generate a correlation Gaussian random process, and then use the random sequence which has the required probability density function for modulation. This method is allowed to independently control the edge of the clutter probability density function and autocorrelation function, and it can be used to generate coherent non-Gaussian correlated radar clutter. The block diagram of SIRP is shown in Fig. 1.

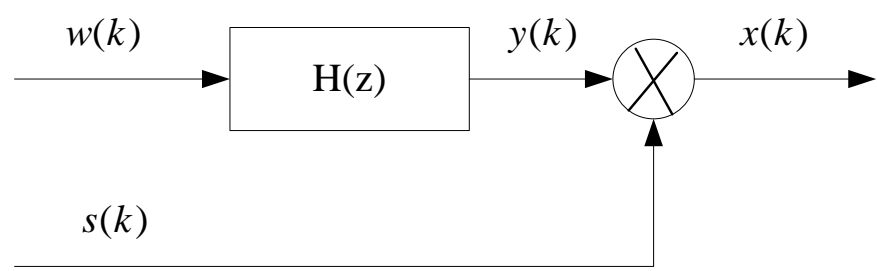

Fig. 1 Process of generating random signal by SIRP 
Complex envelope is $x(k)=y(k) s(k)$, among them $y(k)$ is zero mean multiple correlation Gaussian sequence, $s(k)$ and $y(k)$ are both stationary sequences that are independent with each other, the solution correlation time of $s(k)$ is far longer than $y(k)$. The characteristics of the model is that it can independently control the amplitude distribution and correlation characteristics of $x(k)$. The pdf of amplitude envelope $A(k)=s(k)|y(k)|$ of the complex sequence $x(k)$ is

$$
f_{A}(u, k)=\int_{0}^{\infty} \frac{u}{s^{2} \sigma^{2}(k)} \exp \left(-\frac{u^{2}}{2 s^{2} \sigma^{2}(k)}\right) f(s) d s
$$

It can be seen from the formula that amplitude distribution can be only determined by marginal distribution $f(s)$ of the modulation process $s(k)$.

The complex autocorrelation function of $x(k)$ can be expressed as

$$
r_{x}(n, m)=r_{s}(m) r_{y}(n, m)
$$

As $s(k)$ has a very long correlation time, $r_{y}(m) \approx 1$, the generated autocorrelation function of sequence $x(k)$ is equal to the autocorrelation function of complex correlation Gaussian sequence.

We should take notice of that not all the amplitude distribution can be generated in this way. For a process $x(k)$ that has the amplitude of distribution $f_{A}(u, k)$, we need a marginal distribution $f(s), s \geq 0$. SIRP model can be considered as the degradation of external model.

\section{Mathematical Model for K Distribution}

$\mathrm{K}$ distribution is applicable to describe the heterogeneous clutter of high resolution radar. $\mathrm{K}$ distribution can be expressed by a Rayleigh distribution which has a slowly changing mean, and the slowly changing mean obey $\Gamma$ distribution. The probability density function of K distribution is

$$
f(x)=\frac{2}{a \Gamma(v+1)}\left(\frac{x}{2 a}\right)^{v+1} K_{v}\left(\frac{x}{a}\right) \quad(x>0, v>-1, a>0)
$$

In the formula above, $v$ is the shape parameter and $a$ is the scale parameter. The relationship between $v$ and $a$ can be expressed as

$$
a^{2}=\frac{\sigma^{2}}{2 v}
$$

For most of the clutter, the range of the value of shape parameter is $0<v<\infty$, for the $v$ that has smaller values, for example $v \rightarrow 0.1$, the clutter has a long tail, when $v \rightarrow \infty$ the distribution is close to Rayleigh distribution. Fig. 2 is the block diagram of generating $\mathrm{K}$ distribution clutter.

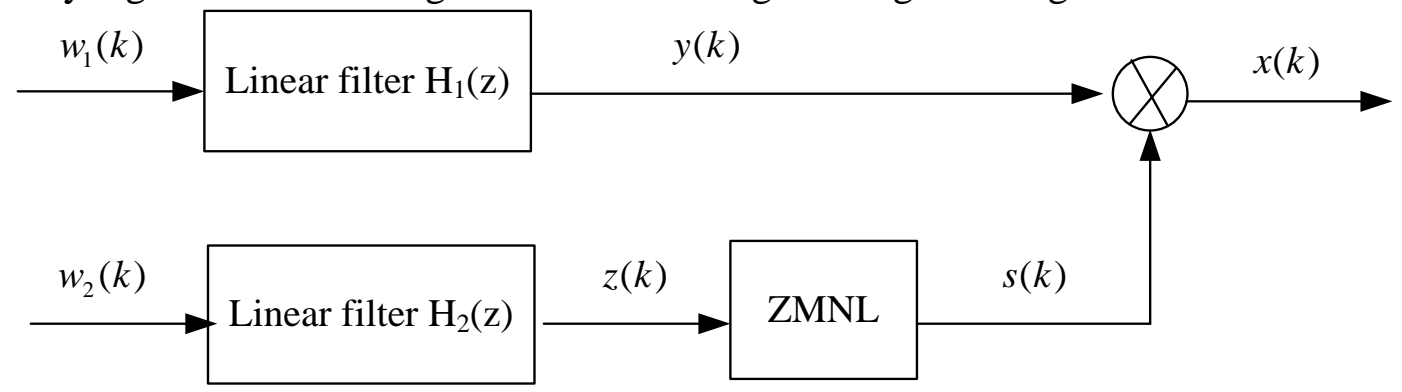

Fig. 2 Generation of K distribution clutter

In Fig. 2, $w_{1}(k)$ is a complex Gaussian white noise, $w_{2}(k)$ is a real Gaussian noise which is independent to $w_{1}(k)$. For $\mathrm{K}$ distribution, which obeys the generalized $\chi$ distribution, the probability density function of the distribution is defined as follows

$$
f(x)=\frac{2 v^{v} x^{2 v-1}}{\Gamma(v)} \exp \left(-v x^{2}\right), \quad x \geq 0
$$

After designing filter $H_{1}(z)$ and $H_{2}(z)$, we can obtain $K$ distribution clutter. 


\section{Simulations}

In this section, we will compare the generated clutter with the theoretical value. Simulation conditions for $\mathrm{K}$ distribution are as follows. The number of radar echo frames is 2000. Pulse repetition frequency is 1000 . Wavelength is 0.05 . Root mean square value is 1.0 . Simulation results are in Fig. 3 and Fig. 4.
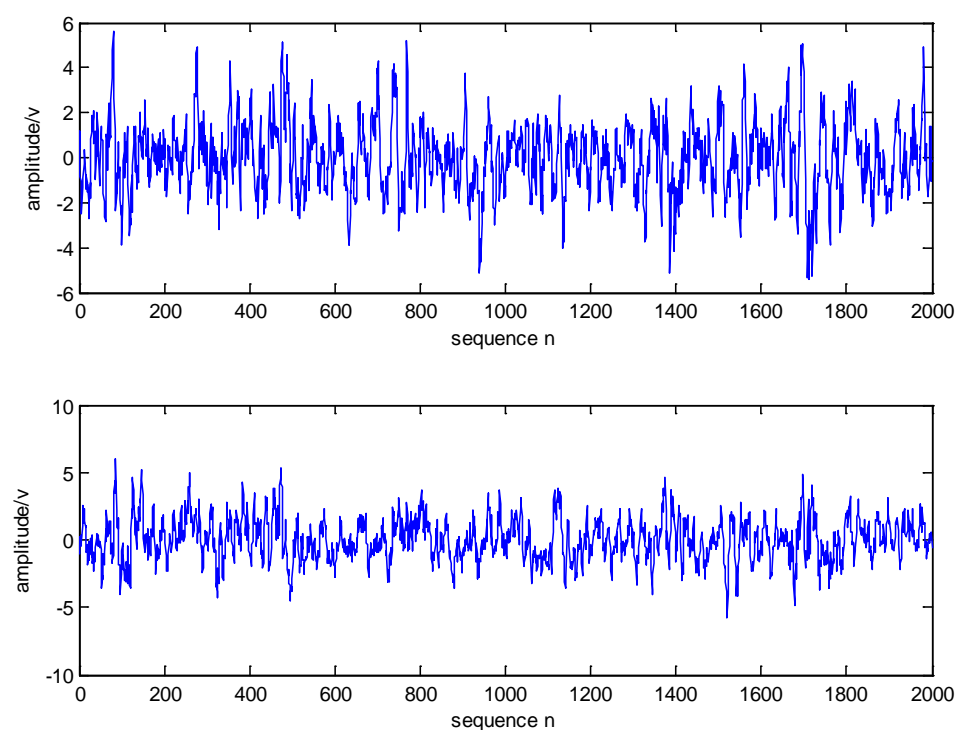

Fig. 3 Real and imaginary part of time domain waveform

Fig. 3 is real and imaginary part of time domain waveform. Fig. 4 is comparision between estimated value and theoretical value. It shows that whether the distribution of clutter amplitude, or the clutter spectrum, the estimated value approaches very near to the theoretical value. The error is mainly because the required Gaussian white noise that has zero mean unit power can not be obtained accurately. The simulation results show that the proposed method is valid.
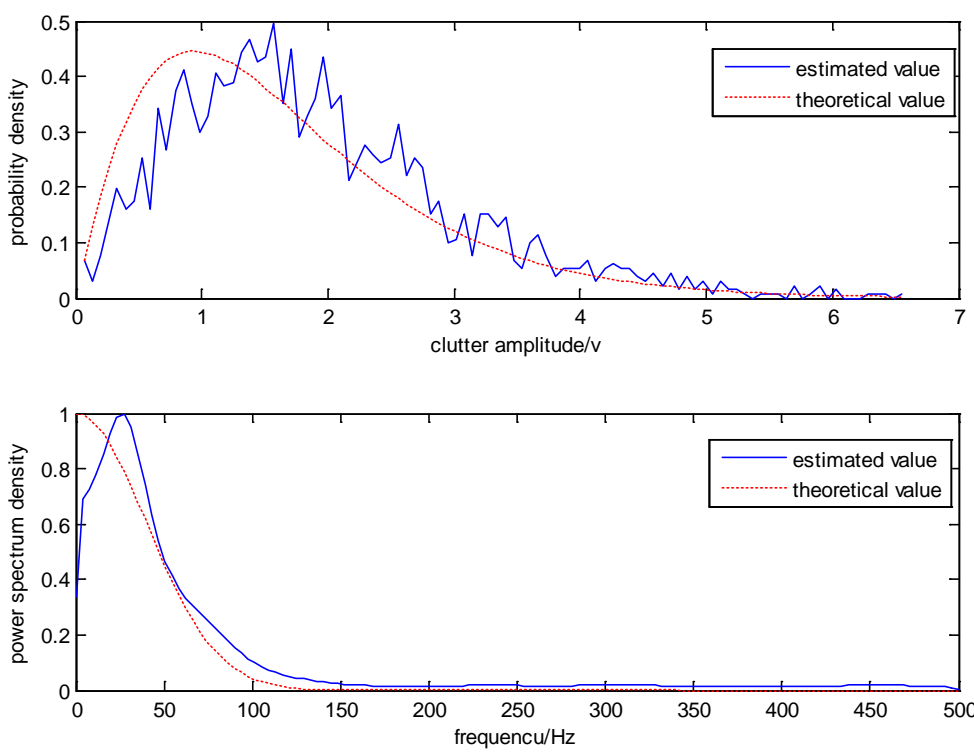

Fig. 4 Comparision between estimated value and theoretical value

\section{Conclusions}

How to model and analyze clutter is very important in the research field of radar. Especially with the development of space technology, electromagnetic environment is becoming more and more complex. So radar is developed towards intelligent direction and clutter modeling needs to be more 
accurate. In this paper, we mainly research on mathematical model for K distribution clutter based on SIRP principle. Simulation results prove the validity of our method.

\section{Acknowledgements}

This work was financially supported by the National Natural Science Foundation of China (61403067), the Program for New Century Excellent Talents in University (NCET-12-0103) and the Natural Science Foundation of Hebei Province (F2013501075).

\section{References}

[1] S. Haykin, Cognitive radar: a way of the future, IEEE Signal Processing Magazine, vol. 23, no. 1(2006), p. 30-40.

[2] A. Karimian and C. Yardim, Multiple grazing sngle sea clutter modeling, IEEE Antennas and Propagation, vol. 60, no. 9(2012), p. 4408-4417.

[3] B. O'Donnell, R. LeBaron and R. Diaz, Physics-based sea clutter model for improved detection of low radar cross-section targets, in Proceedings of IEEE ICASSP, Florence(2014), p. 6830-6833.

[4] R. Duan and X.G. Wang, C.S. Jiang and Z.M. Chen, Space-time clutter model for airborne bistatic radar with non-Gaussian statistics, Journal of Systems Engineering and Electronics, vol. 20, no. 2(2009), p. 283-290.

[5] V. Gregers-Hansen and R. Mital, An empirical sea clutter model for low grazing angles, in Proceedings of IEEE Radar Conference, Pasadena, CA(2009), p. 1-5.

[6] M. Greco and P. Stinco, Identification and analysis of sea radar clutter spikes, IET Radar, Sonar \& Navigation, vol. 4, no. 2(2010), p. 239-250.

[7] L. P. Roy and R. V. Kumar, Accurate K-distributed clutter model for scanning radar application, IET Radar, Sonar \& Navigation, vol. 4, no. 2(2010), p. 158-167. 